\title{
Androgen-sensitive hypertension associated with soluble guanylate cyclase alpha1 deficiency is mediated by 20 -HETE
}

\author{
Sara Vandenwijngaert ${ }^{1}$, Ana C Dordea ${ }^{1}$, Victor Garcia ${ }^{2}$, Robert E Tainsh ${ }^{1}$, Daniel I Nathan ${ }^{1}$, Michael J Raher ${ }^{1}$, \\ Kaitlin Allen', Fan Zhang ${ }^{2}$, Wolfgang S Lieb', Sarah Mikelman', Andrew Kirby ${ }^{3}$, Christine Stevens ${ }^{3}$, \\ Robrecht Thoonen ${ }^{1}$, Allyson Hindle ${ }^{1}$, Patrick Y Sips ${ }^{4}$, Rajeev Malhotra ${ }^{1}$, Mark J Daly ${ }^{3}$, Peter Brouckaert ${ }^{5}$, \\ Kenneth D Bloch ${ }^{1}$, Michal Schwartzman², Emmanuel S Buys ${ }^{1 *}$
}

From 7th International Conference on cGMP Generators, Effectors and Therapeutic Implications Trier, Germany. 19-21 June 2015

\section{Background}

Dysregulated nitric oxide (NO) signaling contributes to the pathogenesis of hypertension. Previously, we reported gender- and strain-specific hypertension in mice deficient in the $\alpha 1$-subunit of the NO receptor soluble guanylate cyclase $\left(\mathrm{sGC \alpha} 1^{-1-}\right)$ : male mice on an Sv129/J (S6) but not a C57BL6/J (B6) background are hypertensive.

\section{Methods and results}

Via linkage analysis, we identified a quantitative trait locus (QTL) associated with elevated blood pressure in male sGC $\alpha 1^{-1-}$ S6 mice. This QTL encompasses CYP4a12a, encoding the predominant murine synthase of the vasoconstrictor 20-hydroxyeicosatetraenoic acid (20-HETE). Renal expression of CYP4a12awas strain-, gender-, and testosterone-dependent:CYP4a12a gene expression was higher in male WT and $\mathrm{sGC} \alpha_{1}{ }^{-/} \mathrm{S} 6$ mice than in female $\mathrm{S} 6$ mice, or than in male and female, WT and $\mathrm{sGC}_{1}{ }^{-1-} \mathrm{B} 6$ mice, higher in testosterone-treated $\mathrm{S} 6$ mice than in vehicle-treated S6 mice, and higher in sham-operated S6 mice than orchiectomized S6 mice. Also, 20-HETE levels were higher in renal preglomerular microvessels of male $\mathrm{sGC} \alpha 1^{-1-}$ S6 than of sGC $\alpha 1^{-1-}$ B6 mice. Furthermore, the 20-HETE antagonist 20-6,15-HEDGE lowered blood pressure in male sGC $\alpha_{1}{ }^{-1-}$ S6 but not WT mice. Finally, the more significant impairment of acetylcholine-induced relaxation of renal interlobar arteries in male $\mathrm{sGC}_{1}{ }^{-1-} \mathrm{S} 6$

\footnotetext{
* Correspondence: ebuys@mgh.harvard.edu

'Department of Anesthesia, Critical Care, and Pain Medicine, Massachusetts General Hospital and Harvard Medical School, Boston, MA 02114, USA Full list of author information is available at the end of the article
}

than $\mathrm{sGC \alpha}{ }_{1}^{-/-} \mathrm{B} 6$ mice, in male $\mathrm{sGC} \alpha_{1}^{-1-} \mathrm{S} 6$ than WT S6 mice, and in male sham-operated $\mathrm{sGC}_{1}{ }^{-1-} \mathrm{S} 6$ mice than orchiectomized sGC $\mathrm{s}_{1}^{-1-} \mathrm{S} 6$ mice was rescued by 20-6,15HEDGE.

\section{Conclusion}

Gender- and strain-specific hypertension and vascular dysfunction in sGC $\alpha 1^{-1-}$ S6 mice is associated with elevated CYP4a $12 a$ expression and 20-HETE levels, and is abrogated by antagonizing 20 -HETE. These results corroborate our hypothesis that testosterone-induced CYP4a12a expression and a concomitant increase in 20-HETE production contribute to the hypertension associated with impaired NO-cGMP signaling and that CYP4a12a represents a candidate blood pressure modifying gene in the context of deficient NO-sGC signaling.

\section{Authors' details \\ ${ }^{1}$ Department of Anesthesia, Critical Care, and Pain Medicine, Massachusetts General Hospital and Harvard Medical School, Boston, MA 02114, USA. 2Department of Pharmacology, New York Medical College, Valhalla, NY 10595, USA. ${ }^{3}$ Center for Human Genetic Research, Department of Medicine, Massachusetts General Hospital, Harvard Medical School, Boston, MA 02114, USA. ${ }^{4}$ Division of Cardiovascular Medicine, Department of Medicine, Brigham and Women's Hospital, Boston, MA 02115, USA. ${ }^{5}$ Department for Biomedical Molecular Biology, Ghent University, Ghent, 9000, Belgium.}

Published: 2 September 2015

\section{doi:10.1186/2050-6511-16-S1-A97}

Cite this article as: Vandenwijngaert et al:: Androgen-sensitive hypertension associated with soluble guanylate cyclase alpha1 deficiency is mediated by 20-HETE. BMC Pharmacology and Toxicology 2015 16(Suppl 1):A97 